\title{
Canadian Labour Archives: Some Recent Acquisitions
}

\author{
Gregory S. Kealey
}

Dalhousie University

The following list is selected from a longer version compiled by Nancy Stunden and published in Labour/LeTravailleur 5 (1980). It is drawn from entries in the 1977-78 Supplement to the Union List of Manuscripts in Canadian Repositories. Lists of earlier acquisitions are contained in Bulletin of the Committee on Canadian Labour History (1976-1979) and in Archivaria 4 (1977).

Public Archives of Canada (Ottawa):

Canadian Labour Congress (additional)

files $1960 \mathrm{~s}$ and $1970 \mathrm{~s} .40 .35 \mathrm{~m}$.

Canadian Railway Labour Association correspondence, etc., c. 1940-70. 5.8m.

Canadian Union of Public Employees files c. $1944-76$, c. $22.3 \mathrm{~cm}$.

Cohen, J.L. (1897-1950)

files and documents of important Toronto Labour and civil rights lawyer. $10.5 \mathrm{~m}$.

Finnish Organization of Canada records $1891-1976,29 \mathrm{~m}$.

International Association of Machinists

Canadian Office Records, 1901-76, 19m

Local Lodge Records, $1908-67,90 \mathrm{~cm}$.

Labour Council of Metropolitan Toronto (additional)

files, 1956-71, 7.8m

Ontario Federation of Labour

files, minutes, reports, $1946-76,10 \mathrm{~m}$.

Ottawa and District Labour Council

minutes, 1899-1968, 5.6m 
Parent-Rowley

files of important textile organizers, 1939-69, 3.4m

Textile Workers Union of America (Canada)

National Office Records 1948-77, 20m.

South-Western Ontario and Toronto Joint Boards, c.1945-67, c.14m.

United Steelworkers of America (Canada)

National Office Records, 1902-76, 17.4m

United Transportation Union (est. 1969)

Brotherhood of Locomotive Firemen, 1886-1974, 25.9m.

Brotherhood of Railway Trainmen, 1897-1971, 4.25m.

Dalhousie University Archives (Halifax, N.S.):

Bell, James K. records, $1947-63,18 \mathrm{~cm}$.

Fillmore, Roscoe member SPC and CPC, 1909-68, 30cm.

Nova Scotia Government Employees Association files and records, 1956-77, 8.5m.

Nova Scotia Labour Relations Board files and records, $1950-79,2.7 \mathrm{~m}$.

Oil, Chemical and Atomic Workers International Union Local 9-823, Pugwash, N. S. minutes, 1937-68, mfm. Local 9-825, Halifax, N.S. minutes, 1942-72, $4 \mathrm{~cm}$.

Provincial Workmen's Association Holdfast Lodge, Joggins, N. S., minutes, 1894-1904. Pioneer Lodge, Springhill, N. S., misc. 1887-1906.

United Mine Workers of America

Local 4514, Springhill, N. S. misc., 1919-1958, 1.3m.

Local 5660, Joggins, N. S. minutes, 1937-40, 121 pp.

United Automobile Workers of America

Local 720, Halifax, minutes, 1963-76, 36m.

Beaton Institute, College of Cape Breton (Sydney, N. S.):

Canadian Labour Congress

Cape Breton Regional Office, records, 1941-75, 7.5m.

Provincial Archives of New Brunswick (Fredericton, N. B.):

United Mine Workers of America

Local 7409, Minto, N. B. 1946-70. 
Archives of Ontario (Toronto):

Thompson, Phillips (1843-1933)

Labour relations consultant and trade unionist, files, 1919-1971, $1.35 \mathrm{~m}$.

Multicultural History Society of Ontario (Toronto):

Finnish Canadian Historical Society Collection

International Ladies Garment Workers Union, Toronto

Toronto Locals and joint board, minutes, 1928-70, 16 reels $\mathrm{mfm}$.

York University Archives (Toronto):

Hodges, Oliver (b.1915)

Labour leader and CCF member, 1943-65, 18.75m.

University of Toronto, Thomas Fisher Rare Book Library:

Kenny, Robert S. Collection

member of the CPC, and collector of left-wing materials, 1920s-1970s, $8.4 \mathrm{~m}$.

McMaster University, Mills Memorial Library Special Collections (Hamilton):

Amalgamated Transit Union

Local 107, Hamilton, minutes, 1899-1972, 3.6m.

Bricklayers, Masons and Plasterers International Union

Local 1, Hamilton, minutes, 1854-1970, 1.8m.

Hamilton and District Labour Council minutes, correspondence, 1886, 96, 1936-70, 22.86m.

Service Employees International Union

Composite local, Toronto, 1944-73, 32.1m.

United Steelworkers of America

District 6, Ontario + West, 1953-72, 18.75m.

Local 1005, Hamilton, 1937-72, 15.5m.

Local 2868, Hamilton, 1943-77, 2.5m.

Saskatchewan Archives Board:

Canadian Union of Public Employees

Saskatchewan Region, minutes, 1945-75, 10.5m.

International Typographical Union

Local 663, Saskatoon, minutes, 1906-56, reel

Regina Trades and Labour Council

minutes, $2 \mathrm{~m}$. 
Saskatoon Labour Council

minutes, 1945-53 and 1956-62, 1.2m.

Service Employees International Union

Local 333, Saskatoon, minutes, 1946-74, 5.7m.

Regional Council No.15, Saskatoon, minutes, 1957-72, 3.15m.

Glenbow-Alberta Institute Archives:

Calgary Trades and Labour Council

minutes, 1913-28 and 1947-52, 3m.

United Mineworkers of America

District 18, minutes, 1903-64, 3.96m.

University of British Columbia Library, Special Collections:

British Columbia Federation of Labour

files, 1960-73, $25 \mathrm{~m}$.

International Woodworkers of America

District Council 1, minutes, 1931-48, 1.8m.

International Association of Bridge, Structural and Ornamental Iron Workers Local 712, Burnaby, minutes, 1947-70, 10.5m.

City Archives of Vancouver:

Vancouver Typographical Union minutes, $1884-1970,1.28 \mathrm{~m}$.

Provincial Archives of British Columbia:

International Union of Mine, Mill and Smelter Workers

Local 98, Slocan Lake, minutes 1912-24, 4m.

Murphy, Harvey

correspondence, etc, 1942-73, 10.6m. 